Also available at http://amc.imfm.si

ISSN 1855-3966 (printed edn.), ISSN 1855-3974 (electronic edn.)

ARS MATHEMATICA CONTEMPORANEA 6 (2013) 289-299

\title{
GCD-Graphs and NEPS of Complete Graphs
}

\author{
Walter Klotz \\ Institut für Mathematik, Technische Universität Clausthal, Germany \\ Torsten Sander
}

Fakultät für Informatik, Ostfalia Hochschule für angewandte Wissenschaften, Germany

Received 21 February 2012, accepted 20 August 2012, published online 19 November 2012

\begin{abstract}
A gcd-graph is a Cayley graph over a finite abelian group defined by greatest common divisors. Such graphs are known to have integral spectrum. A non-complete extended psum, or NEPS in short, is well-known general graph product. We show that the class of gcd-graphs and the class of NEPS of complete graphs coincide. Thus, a relation between the algebraically defined Cayley graphs and the combinatorially defined NEPS of complete graphs is established. We use this link to show that gcd-graphs have a particularly simple eigenspace structure, to be precise, that every eigenspace of the adjacency matrix of a gcdgraph has a basis with entries $-1,0,1$ only.
\end{abstract}

Keywords: Integral graphs, Cayley graphs, graph products.

Math. Subj. Class.: 05C25, 05C50

\section{Introduction}

Given a set $B \subseteq\{0,1\}^{n}$ and graphs $G_{1}, \ldots, G_{n}$, the NEPS (non-complete extended psum) of these graphs with respect to basis $B, G=\operatorname{NEPS}\left(G_{1}, \ldots, G_{n} ; B\right)$, has as its vertex set the Cartesian product of the vertex sets of the individual graphs, $V(G)=V\left(G_{1}\right) \times \cdots \times$ $V\left(G_{n}\right)$. Distinct vertices $x=\left(x_{1}, \ldots, x_{n}\right), y=\left(y_{1}, \ldots, y_{n}\right) \in V(G)$ are adjacent in $G$, if and only if there exists some n-tuple $\left(\beta_{1}, \ldots, \beta_{n}\right) \in B$ such that $x_{i}=y_{i}$, whenever $\beta_{i}=0$, and $x_{i}, y_{i}$ are distinct and adjacent in $G_{i}$, whenever $\beta_{i}=1$. In particular, $\operatorname{NEPS}\left(G_{1} ;\{(1)\}\right)=G_{1}$ and $\operatorname{NEPS}\left(G_{1} ; \emptyset\right)=\operatorname{NEPS}\left(G_{1} ;\{(0)\}\right)$ is the graph without edges on the vertices of $G_{1}$.

The NEPS operation generalizes a number of known graph products, all of which have in common that the vertex set of the resulting graph is the Cartesian product of the input vertex sets. For example, $\operatorname{NEPS}\left(G_{1}, \ldots, G_{n} ;\{(1,1, \ldots, 1)\}\right)=G_{1} \otimes \ldots \otimes G_{n}$ is the

E-mail addresses: klotz@math.tu-clausthal.de (Walter Klotz), t.sander@ostfalia.de (Torsten Sander) 
product of $G_{1}, \ldots, G_{n}$ (cf. [10], "direct product" in [15]). As can be seen, unfortunately, the naming of graph products is not standardized at all. The "Cartesian product" of graphs in [15] is even known as the "sum" of graphs in [10]. With respect to this seemingly arbitrary mixing of sum and product terminology, let us point out that here the term "sum" (and also the "p-sum" contained in the NEPS acronym) indicates that the adjacency matrix of the constructed product graph arises from a certain sum of matrices (involving the adjacency matrices of the input graphs). Refer to [10] or [11] for the history of the notion of NEPS. We remark that the NEPS operation can be generalized even further, see e.g. [12] and [21].

Next, we consider the important class of Cayley graphs [13]. These graphs have been and still are studied intensively because of their symmetry properties and their connections to communication networks, quantum physics and other areas [8], [13]. Let $\Gamma$ be a finite, additive group. A subset $S \subseteq \Gamma$ is called a symbol (also: connection set, shift set) of $\Gamma$ if $-S=\{-s: s \in S\}=S, 0 \notin S$. The undirected Cayley graph over $\Gamma$ with symbol $S$, denoted by $\operatorname{Cay}(\Gamma, S)$, has vertex set $\Gamma$; two vertices $a, b \in \Gamma$ are adjacent if and only if $a-b \in S$.

Let us now construct the class of ged-graphs. The greatest common divisor of nonnegative integers $a$ and $b$ is denoted by $\operatorname{gcd}(a, b), \operatorname{gcd}(0, b)=\operatorname{gcd}(b, 0)=b$. If $x=$ $\left(x_{1}, \ldots, x_{r}\right)$ and $m=\left(m_{1}, \ldots, m_{r}\right)$ are tuples of nonnegative integers, then we set

$$
\operatorname{gcd}(x, m)=\left(d_{1}, \ldots, d_{r}\right)=d, d_{i}=\operatorname{gcd}\left(x_{i}, m_{i}\right) \text { for } i=1, \ldots, r .
$$

For an integer $n \geq 1$ we denote by $Z_{n}$ the additive group of integers modulo $n$, the ring of integers modulo $n$, or simply the set $\{0,1, \ldots, n-1\}$. The particular choice will be clear from the context. Let $\Gamma$ be an (additive) finite abelian group represented as a direct sum of cyclic groups,

$$
\Gamma=Z_{m_{1}} \oplus \ldots \oplus Z_{m_{r}}, m_{i} \geq 1 \text { for } i=1, \ldots, r .
$$

Suppose that $d_{i}$ is a divisor of $m_{i}, 1 \leq d_{i} \leq m_{i}$, for $i=1, \ldots, r$. For the divisor tuple $d=\left(d_{1}, \ldots, d_{r}\right)$ of $m=\left(m_{1}, \ldots, m_{r}\right)$ we define

$$
S_{\Gamma}(d)=\left\{x=\left(x_{1}, \ldots, x_{r}\right) \in \Gamma: \operatorname{gcd}(x, m)=d\right\} .
$$

Let $D=\left\{d^{(1)}, \ldots, d^{(k)}\right\}$ be a set of distinct divisor tuples of $m$ and define

$$
S_{\Gamma}(D)=\bigcup_{j=1}^{k} S_{\Gamma}\left(d^{(j)}\right) .
$$

Observe that the union is actually disjoint. The sets $S_{\Gamma}(D)$ shall be called gcd-sets of $\Gamma$. We define the class of $g c d$-graphs as the Cayley graphs Cay $(\Gamma, S)$ over a finite abelian group $\Gamma$ with symbol $S$ a gcd-set of $\Gamma$. The most prominent members of this class are perhaps the unitary Cayley graphs $X_{n}=\operatorname{Cay}\left(Z_{n}, U_{n}\right)$, where $U_{n}=S_{Z_{n}}(1)$ is the multiplicative group of units of $Z_{n}$ (cf. [16], [17], [22]).

The main goal of this paper is to show in Section 2 that every gcd-graph is isomorphic to a NEPS of complete graphs. Conversely, every NEPS of complete graphs is isomorphic to a gcd-graph over some abelian group. This relation is remarkable since it allows us to define gcd-graphs either algebraically (via Cayley graphs) or purely combinatorially (via NEPS). The characterization of gcd-graphs as NEPS of complete graphs reveals some new access to structural properties of ged-graphs. As a first application, we show in Section 
3 that every gcd-graph has simply structured eigenspace bases for all of its eigenvalues. This means that for every eigenspace a basis can be found whose vectors only have entries from the set $\{0,1,-1\}$. It is known that other graph classes exhibit a similar eigenspace structure, although not necessarily for all of their eigenspaces [9], [20], [25]. Finally, we present some open problems in Section 4.

\section{Isomorphisms between NEPS of complete graphs and ged-graphs}

We are going to show in several steps that ged-graphs and NEPS of complete graphs are the same.

Lemma 2.1. Let $\Gamma=Z_{m_{1}} \oplus \cdots \oplus Z_{m_{r}}$ and $d=\left(d_{1}, \ldots, d_{r}\right)$ a tuple of positive divisors of $m=\left(m_{1}, \ldots, m_{r}\right)$. Define $b=\left(b_{i}\right) \in\{0,1\}^{r}$ by

$$
b_{i}= \begin{cases}1 & \text { if } d_{i}<m_{i} \\ 0 & \text { if } d_{i}=m_{i}\end{cases}
$$

Then we have

$$
\operatorname{Cay}\left(\Gamma, S_{\Gamma}(d)\right)=\operatorname{NEPS}\left(\operatorname{Cay}\left(Z_{m_{1}}, S_{Z_{m_{1}}}\left(d_{1}\right)\right), \ldots, \operatorname{Cay}\left(Z_{m_{r}}, S_{Z_{m_{r}}}\left(d_{r}\right)\right) ;\{b\}\right) .
$$

Proof. Both $\operatorname{Cay}\left(\Gamma, S_{\Gamma}(d)\right)$ and the above NEPS have the same vertex set $\Gamma$. It remains to show that they have the same edge set.

Let $x, y \in \Gamma$ with $x=\left(x_{1}, \ldots, x_{r}\right), y=\left(y_{1}, \ldots, y_{r}\right)$ and suppose that $x \neq y$. Now $x$ and $y$ are adjacent in $\operatorname{Cay}\left(\Gamma, S_{\Gamma}(d)\right)$ if and only if $\operatorname{gcd}\left(x_{i}-y_{i}, m_{i}\right)=d_{i}$ for $i=1, \ldots, r$. The latter condition means that in case $d_{i}<m_{i}$ the vertices $x_{i}$ and $y_{i}$ are adjacent in $G_{i}=\operatorname{Cay}\left(Z_{m_{i}}, S_{Z_{m_{i}}}\left(d_{i}\right)\right)$, and in case $d_{i}=m_{i}$ we have $x_{i}=y_{i}$. But this is exactly the condition for adjacency of $x$ and $y$ in $\operatorname{NEPS}\left(G_{1}, \ldots, G_{r} ;\{b\}\right)$.

The following lemma allows us to break down the Cayley graphs that form the factors of the NEPS mentioned in Lemma 2.1. Each factor can be transformed into a gcd-graph over a product of cyclic groups of prime power order. Using Lemma 2.1 once again, we obtain a representation of the original graph as a NEPS of NEPS of gcd-graphs over cyclic groups of prime power order.

Lemma 2.2. Let the integer $m \geq 2$ and a proper divisor $d \geq 1$ of $m$ be given as products of powers of distinct primes,

$$
\begin{aligned}
& m=\prod_{i=1}^{r} m_{i}, \quad m_{i}=p_{i}^{\alpha_{i}}, \alpha_{i}>0 \text { for } i=1, \ldots, r \\
& d=\prod_{i=1}^{r} d_{i}, \quad d_{i}=p_{i}^{\beta_{i}}, 0 \leq \beta_{i} \leq \alpha_{i} \text { for } i=1, \ldots, r .
\end{aligned}
$$

If we set $\Gamma=Z_{m_{1}} \oplus \cdots \oplus Z_{m_{r}}$ and $\tilde{d}=\left(d_{1}, \ldots, d_{r}\right)$, then there exists an isomorphism

$$
\operatorname{Cay}\left(Z_{m}, S_{Z_{m}}(d)\right) \simeq \operatorname{Cay}\left(\Gamma, S_{\Gamma}(\tilde{d})\right)
$$


Proof. By the Chinese remainder theorem [23] we know that every $z \in Z_{m}$ is uniquely determined by the congruences

$$
z \equiv z_{i} \bmod m_{i}, z_{i} \in Z_{m_{i}} \text { for } i=1, \ldots, r .
$$

This gives rise to a bijection $Z_{m} \rightarrow \Gamma$ by virtue of $z \mapsto\left(z_{1}, \ldots, z_{r}\right)=: \tilde{z}$. We show that this bijection induces an isomorphism between $\operatorname{Cay}\left(Z_{m}, S_{Z_{m}}(d)\right)$ and $\operatorname{Cay}\left(\Gamma, S_{\Gamma}(\tilde{d})\right)$.

Let $x, y \in Z_{m}, x \neq y$. Note that $\tilde{x}$ and $\tilde{y}$ are vertices of Cay $\left(\Gamma, S_{\Gamma}(\tilde{d})\right)$. The vertices $x$ and $y$ are adjacent in $\operatorname{Cay}\left(Z_{m}, S_{Z_{m}}(d)\right)$ if and only if $\operatorname{gcd}(x-y, m)=d$. This is equivalent to $\operatorname{gcd}\left(x_{i}-y_{i}, m_{i}\right)=d_{i}$ for every $i=1, \ldots, r$. Now this means $\operatorname{gcd}(\tilde{x}-\tilde{y}, \tilde{m})=\tilde{d}$, with $\tilde{m}=\left(m_{1}, \ldots, m_{r}\right)$, which is the condition for adjacency of $\tilde{x}$ and $\tilde{y}$ in $\operatorname{Cay}\left(\Gamma, S_{\Gamma}(\tilde{d})\right)$.

Next we shall prove a lemma that helps us consolidate the nesting of NEPS operations into a single NEPS operation. As a result, we then know that every single-divisor tuple gcd-graph is isomorphic to a NEPS of gcd-graphs over cyclic groups of prime power order.

Lemma 2.3. Let

$$
H=\operatorname{NEPS}\left(H^{(1)}, \ldots, H^{(t)} ; B\right)
$$

be a NEPS of graphs $H^{(j)}$ with respect to basis $B$ such that each graph $H^{(j)}$ is itself a NEPS of graphs $G_{i}^{(j)}$ with respect to basis $B^{(j)}$,

$$
H^{(j)}=\operatorname{NEPS}\left(G_{1}^{(j)}, \ldots, G_{r_{j}}^{(j)} ; B^{(j)}\right) \text { for } j=1, \ldots, t .
$$

Then there exists a set $B^{\prime} \subseteq\{0,1\}^{r}, r=r_{1}+\ldots+r_{t}$, such that

$$
H \simeq \operatorname{NEPS}\left(G_{1}^{(1)}, \ldots, G_{r_{1}}^{(1)}, \ldots, G_{1}^{(t)}, \ldots, G_{r_{t}}^{(t)} ; B^{\prime}\right) .
$$

Proof. We show that in $(2.1)$ the graph $H^{(1)}$ can be replaced by $G_{1}^{(1)}, \ldots, G_{r_{1}}^{(1)}$. More precisely, we construct a set $\tilde{B}$ such that

$$
H \simeq \operatorname{NEPS}\left(G_{1}^{(1)}, \ldots, G_{r_{1}}^{(1)}, H^{(2)}, \ldots, H^{(t)} ; \tilde{B}\right), \quad \tilde{B} \subseteq\{0,1\}^{r_{1}+t-1} .
$$

An analogous procedure can be repeated for $H^{(2)}, \ldots, H^{(t)}$ until we end up with the representation (2.3) of $H$.

In the original representation (2.1) every vertex $x$ of the vertex set $V(H)$ has the form

$$
x=\left(x^{(1)}, \ldots, x^{(t)}\right), x^{(j)} \in V\left(H^{(j)}\right) \text { for } j=1, \ldots, t .
$$

By (2.2) each coordinate $x^{(j)}$ is itself an $r_{j}$-tuple, in particular

$$
x^{(1)}=\left(x_{1}^{(1)}, \ldots, x_{r_{1}}^{(1)}\right), x_{i}^{(1)} \in V\left(G_{i}^{(1)}\right) \text { for } i=1, \ldots, r_{1} .
$$

Expansion of $x^{(1)}$ in (2.5) yields

$$
\begin{aligned}
& \tilde{x}=\left(x_{1}^{(1)}, \ldots, x_{r_{1}}^{(1)}, x^{(2)}, \ldots, x^{(t)}\right) \\
& x_{i}^{(1)} \in V\left(G_{i}^{(1)}\right) \text { for } i=1, \ldots, r_{1}, x^{(j)} \in V\left(H^{(j)}\right) \text { for } j=2, \ldots, t .
\end{aligned}
$$

This is the representation of vertices for (2.4). 
Now we adapt the basis set $B$ to the new representation of vertices of $H$ such that adjacencies remain unchanged. Let the distinct vertices $x$ and $y$ of $H$ be given in their original representation according to (2.5) and in their new representation $\tilde{x}, \tilde{y}$ according to (2.6).

$$
\begin{aligned}
& x=\left(x^{(1)}, \ldots, x^{(t)}\right), y=\left(y^{(1)}, \ldots, y^{(t)}\right) \\
& \tilde{x}=\left(x_{1}^{(1)}, \ldots, x_{r_{1}}^{(1)}, x^{(2)}, \ldots, x^{(t)}\right), \tilde{y}=\left(y_{1}^{(1)}, \ldots, y_{r_{1}}^{(1)}, y^{(2)}, \ldots, y^{(t)}\right) .
\end{aligned}
$$

For each $b=\left(b_{1}, \ldots, b_{t}\right) \in B$ we define a set $\tilde{B}(b) \subseteq\{0,1\}^{r_{1}+t-1}$ such that

$x, y$ adjacent with respect to $b \Leftrightarrow \tilde{x}, \tilde{y}$ adjacent with respect to $\tilde{B}(b)$.

Case 1: $b_{1}=0$.

For $x$ and $y$ to be adjacent with respect to $b$ we must have $x^{(1)}=y^{(1)}$. If this is satisfied, then $x$ and $y$ are adjacent, if and only if $\left(x^{(2)}, \ldots, x^{(t)}\right)$ and $\left(y^{(2)}, \ldots, y^{(t)}\right)$ are adjacent with respect to $\left(b_{2}, \ldots, b_{t}\right)$. We achieve $(2.7)$ by setting $\tilde{b}=\left(0, \ldots, 0, b_{2}, \ldots, b_{t}\right)$ (first $r_{1}$ entries equal to zero) and $\tilde{B}(b)=\{\tilde{b}\}$.

Case 2: $b_{1}=1$.

Now $x$ and $y$ are adjacent with respect to $b$, if and only if $x^{(1)}$ and $y^{(1)}$ are adjacent in $H^{(1)}$ and $x^{(2)}, \ldots, x^{(t)}$ and $y^{(2)}, \ldots, y^{(t)}$ are equal or adjacent with respect to $b_{2}, \ldots, b_{t}$, respectively. By (2.2) vertices $x^{(1)}$ and $y^{(1)}$ of $H^{(1)}$ are adjacent, if and only if they are adjacent with respect to some $b^{(1)}=\left(b_{1}^{(1)}, \ldots, b_{r_{1}}^{(1)}\right) \in B^{(1)}$. In this case we satisfy (2.7) by setting

$$
\tilde{B}(b)=\left\{\left(b_{1}^{(1)}, \ldots, b_{r_{1}}^{(1)}, b^{(2)}, \ldots, b^{(t)}\right): b^{(1)} \in B^{(1)}\right\} .
$$

Finally, we collect the new basis tuples in $\tilde{B}=\cup\{\tilde{B}(b): \quad b \in B\}$ and thus achieve (2.4).

The next step towards our goal is to show that a single-divisor gcd-graph over a cyclic group of prime power order is actually isomorphic to a NEPS of complete graphs.

We denote the complete graph on $n$ vertices by $K_{n}$. For our purposes, we assume that the vertex set of $K_{n}$ is $Z_{n}=\{0,1, \ldots, n-1\}$.

Lemma 2.4. Let $m=p^{\alpha}$ be a prime power, $d=p^{\beta}$ a divisor of $m, 0 \leq \beta \leq \alpha$. Then the gcd-graph over $Z_{m}$ with respect to $d$ is isomorphic to a NEPS of $\alpha$ copies of the complete graph $K_{p}$, i.e.

$$
\operatorname{Cay}\left(Z_{m}, S_{Z_{m}}(d)\right) \simeq \operatorname{NEPS}\left(K_{p}, \ldots, K_{p} ; B\right) \text { for some } B \subseteq\{0,1\}^{\alpha} .
$$

Proof. In case $\beta=\alpha$ we have $\operatorname{Cay}\left(Z_{m}, S_{Z_{m}}(m)\right) \simeq \operatorname{NEPS}\left(K_{p}, \ldots, K_{p} ;\{(0, \ldots, 0)\}\right)$. So we may now assume $\beta<\alpha$.

Let us denote $G=\operatorname{Cay}\left(Z_{m}, S_{Z_{m}}(d)\right)$ and $H=\operatorname{NEPS}\left(K_{p}, \ldots, K_{p} ; B\right)$ (where the basis $B$ is not yet fixed). For every $x \in Z_{m}$ let $\left(x_{0}, \ldots, x_{\alpha-1}\right)$ be defined by the $p$-adic representation of $x$,

$$
x=\sum_{i=0}^{\alpha-1} x_{i} p^{i}, 0 \leq x_{i}<p \text { for } i=0, \ldots, \alpha-1 .
$$


We shall assume that the vertex set of $K_{p}$ is $Z_{p}$. Define the bijection $\varphi: Z_{m} \rightarrow Z_{p} \oplus \cdots \oplus$ $Z_{p}=Z_{p}^{\alpha}$ by $\varphi(x)=\left(x_{0}, \ldots, x_{\alpha-1}\right)$. We now construct a basis set $B \subseteq\{0,1\}^{\alpha}$ such that $\varphi$ induces an isomorphism between $G$ and $H$. Observe that for every $z \in Z_{m}$,

$$
\operatorname{gcd}(z, m)=d \Leftrightarrow z_{i}=0 \text { for every } i<\beta \text { and } z_{\beta} \neq 0 .
$$

This leads to the definition of $B$ as follows:

$$
B=\left\{\left(b_{0}, \ldots, b_{\alpha-1}\right) \in\{0,1\}^{\alpha}: b_{i}=0 \text { for every } i<\beta, b_{\beta}=1\right\} .
$$

Let $x, y \in Z_{m}, x \neq y, \varphi(x)=\left(x_{0}, \ldots, x_{\alpha-1}\right), \varphi(y)=\left(y_{0}, \ldots, y_{\alpha-1}\right)$. Now $x$ and $y$ are adjacent in $G$ if and only if $\operatorname{gcd}(x-y, m)=d$, which means $x_{i}-y_{i}=0$ for every $i<\beta$ and $x_{\beta}-y_{\beta} \neq 0$. Thanks to our choice of $B$, this is exactly the condition for $\varphi(x)$ and $\varphi(y)$ being adjacent in $H$.

Theorem 2.5. Let $G$ be an arbitrary gcd-graph, $G=\operatorname{Cay}\left(\Gamma, S_{\Gamma}(D)\right), \Gamma=Z_{m_{1}} \oplus \cdots \oplus$ $Z_{m_{r}}, D=\left\{d^{(1)}, \ldots, d^{(k)}\right\}$ a set of divisor tuples of $m=\left(m_{1}, \ldots, m_{r}\right)$. If $n=p_{1} \cdots p_{t}$ is the prime factorization of $n=m_{1} \cdots m_{r}$, then

$$
G \simeq \operatorname{NEPS}\left(K_{p_{1}}, \ldots, K_{p_{t}} ; B\right)=H \text { for some } B \subseteq\{0,1\}^{t} .
$$

Proof. Each divisor tuple in $D$ gives rise to a graph $G^{(j)}=\operatorname{Cay}\left(\Gamma, S_{\Gamma}\left(d^{(j)}\right)\right), j=$ $1, \ldots, k$. By application of the preceding lemmas of this section we know that

$$
G^{(j)} \simeq \operatorname{NEPS}\left(K_{p_{1}}, \ldots, K_{p_{t}} ; B^{(j)}\right)=H^{(j)} \text { for some } B^{(j)} \subseteq\{0,1\}^{t} .
$$

The graphs $G^{(j)}$ constitute an edge disjoint decomposition of $G$. Now, for every divisor tuple $d^{(1)}, \ldots, d^{(k)} \in D$, we perform the decomposition process outlined by the lemmas in exactly the same way, in the sense that the vertex numberings of the resulting graphs $H^{(j)}$ are coherent. Then the graphs $H^{(j)}$ also constitute an edge disjoint decomposition of $G$ :

$$
E(G)=\bigcup_{j=1}^{k} E\left(G^{(j)}\right), E(H)=\bigcup_{j=1}^{k} E\left(H^{(j)}\right)
$$

The binary sets $B^{(j)}, 1 \leq j \leq k$, are also pairwise disjoint. The disjoint union of the edge sets $E\left(H^{(j)}\right), 1 \leq j \leq k$, is generated in the NEPS of $K_{p_{1}}, \ldots, K_{p_{t}}$ by

$$
B=\bigcup_{j=1}^{k} B^{(j)}
$$

With this choice of $B$ the isomorphisms between the subgraphs $G^{(j)}$ and $H^{(j)}, 1 \leq j \leq k$, extend to an isomorphism between $G$ and $H$.

Theorem 2.6. Let $G$ be a NEPS of complete graphs, $G=\operatorname{NEPS}\left(K_{m_{1}}, \ldots, K_{m_{r}} ; B\right)$. Then $G$ is isomorphic to a gcd-graph over $\Gamma=Z_{m_{1}} \oplus \cdots \oplus Z_{m_{r}}$.

Proof. The vertex set of $G$ can be represented by $\Gamma=Z_{m_{1}} \oplus \cdots \oplus Z_{m_{r}}$. Edges of $G$ are generated by the binary $r$-tuples $b=\left(b_{i}\right)$ of the basis set $B$. Vertices $x=\left(x_{1}, \ldots, x_{r}\right) \neq$ $y=\left(y_{1}, \ldots, y_{r}\right)$ are adjacent in $G$ with respect to $b$, if $x_{i}=y_{i}$, whenever $b_{i}=0$, and 
$x_{i} \neq y_{i}$, whenever $b_{i}=1$. Let the set $D(b)$ consist of all positive divisor tuples $d=$ $\left(d_{1}, \ldots, d_{r}\right)$ of $m=\left(m_{1}, \ldots, m_{r}\right)$ such that $d_{i}=m_{i}$, whenever $b_{i}=0$, and $d_{i}$ a proper divisor of $m_{i}$, whenever $b_{i}=1$. Then $x$ and $y$ are adjacent with respect to $b$, if and only if $\operatorname{gcd}(x-y, m) \in D(b)$. If we define $D=\cup\{D(b): b \in B\}$, then the gcd-graph $\operatorname{Cay}\left(\Gamma, S_{\Gamma}(D)\right)$ is isomorphic to $G$.

Theorems 2.5 and 2.6 imply the following corollary.

Corollary 2.7. Let $n=p_{1} \cdots p_{t}$ be the prime factorization of the integer $n \geq 2$. Every gcd-graph with $n$ vertices is isomorphic to a gcd-graph over $\Gamma=Z_{p_{1}} \oplus \cdots \oplus Z_{p_{t}}$.

We conclude this section with some examples.

Example 2.8. We generalize the definition of a Hamming graph given in [15]. The Hamming graph $G=\operatorname{Ham}\left(m_{1}, \ldots, m_{r} ; D\right)$ has vertex set $V(G)=Z_{m_{1}} \oplus \ldots \oplus Z_{m_{r}}$. Distinct vertices are adjacent in $G$, if their Hamming distance is in $D$. It can be easily shown that $G$ is a NEPS of the complete graphs $K_{m_{1}}, \ldots, K_{m_{r}}$.

Example 2.9. Sudoku graphs arise from the popular game of Sudoku. The Sudoku graph $\operatorname{Sud}(n)$ models the number restrictions imposed when filling out an $n^{2} \otimes n^{2}$ Sudoku puzzle. Each vertex represents a cell of the Sudoku puzzle. Two vertices are adjacent if the two corresponding cells are required to contain different numbers (which is the case when they lie in the same row, column or block of the puzzle). It has been shown that Sudoku graphs are NEPS of complete graphs [25].

Example 2.10. This is an example that demonstrates the application of Theorem 2.5. Let $\Gamma=Z_{4} \oplus Z_{18}$ and $D=\{(1,6),(4,2),(2,9)\}$. We want to represent the graph $\operatorname{Cay}\left(\Gamma, S_{\Gamma}(D)\right)$ as a NEPS of complete graphs. To start with, let us consider the graph Cay $\left(\Gamma, S_{\Gamma}((1,6))\right)$. Application of Lemma 2.1, Lemma 2.2, once again Lemma 2.1, then Lemma 2.3, Lemma 2.4, and finally once again Lemma 2.3 gives us:

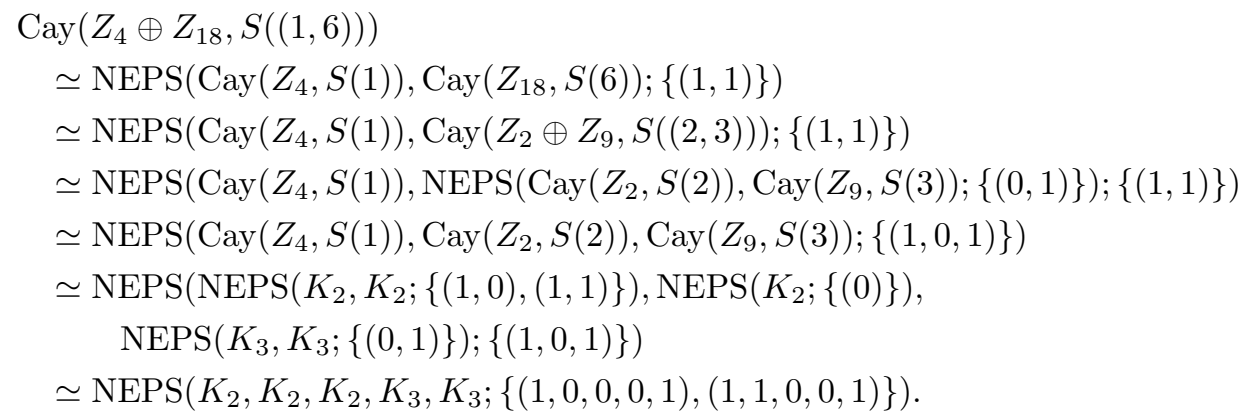

Note that for the sake of simplicity we have dropped the subscripts of the symbol sets since the respective groups are clear from the context. Regarding the application of Lemma 2.3 note that, trivially, $G \simeq \operatorname{NEPS}(G ;\{(1)\})$.

$$
\begin{aligned}
\operatorname{Cay}\left(Z_{4} \oplus Z_{18}, S((4,2))\right) \simeq & \operatorname{NEPS}\left(K_{2}, K_{2}, K_{2}, K_{3}, K_{3} ;\right. \\
& \{(0,0,0,1,0),(0,0,0,1,1)\}), \\
\operatorname{Cay}\left(Z_{4} \oplus Z_{18}, S((2,9))\right) \simeq & \operatorname{NEPS}\left(K_{2}, K_{2}, K_{2}, K_{3}, K_{3} ;\{(0,1,1,0,0)\}\right) .
\end{aligned}
$$


The graph $\operatorname{Cay}\left(\Gamma, S_{\Gamma}(D)\right)$ is the disjoint union of the graphs $\operatorname{Cay}\left(\Gamma, S_{\Gamma}(d)\right)$ with $d \in$ $D$ which we have considered above, so we arrive at:

$$
\begin{array}{cl}
\operatorname{Cay}\left(\Gamma, S_{\Gamma}(D)\right) \simeq \quad & \operatorname{NEPS}\left(K_{2}, K_{2}, K_{2}, K_{3}, K_{3} ;\right. \\
& \{(1,0,0,0,1),(1,1,0,0,1),(0,0,0,1,0), \\
& (0,0,0,1,1),(0,1,1,0,0)\}) .
\end{array}
$$

\section{Eigenspace bases of gcd-graphs}

The eigenvalues and eigenspaces of an undirected graph $G$ are the eigenvalues and eigenspaces, respectively, of any adjacency matrix of $G$. The multiset of all eigenvalues of a graph is called its spectrum. According to HARARY and SCHWENK [14], a graph $G$ is defined to be integral if all of its eigenvalues are integers. Integral graphs have been a focus of research for some time; see [4] for a survey.

In particular, many notable results on integrality of Cayley graphs have been obtained. Integral cubic and quartic Cayley graphs on abelian groups have been characterized in [1] and [2], respectively. Circulant graphs are the Cayley graphs over $Z_{n}, n \geq 1$. So [26] showed that the integral circulant graphs with $n$ vertices are exactly the gcd-graphs over $Z_{n}$. This result was extended in [18] to groups of the form $Z_{2} \oplus \ldots \oplus Z_{2} \oplus Z_{n}, n \geq 2$. A complete characterization of integral Cayley graphs over abelian groups has recently been achieved by ALPERIN and PETERSON [3].

The eigenvalues of $G=\operatorname{NEPS}\left(G_{1}, \ldots, G_{n} ; B\right)$ are certain sums of products of the eigenvalues of the $G_{i}$, cf. [10]:

Theorem 3.1. Let $G_{1}, \ldots, G_{n}$ be graphs with $n_{1}, \ldots, n_{r}$ vertices, respectively. Further, for $i=1, \ldots, r$ let $\lambda_{i 1}, \ldots, \lambda_{i n_{i}}$ be the eigenvalues of $G_{i}$. Then, the spectrum of the graph $G=\operatorname{NEPS}\left(G_{1}, \ldots, G_{n} ; B\right)$ with respect to basis $B$ consist of all possible values

$$
\mu_{i_{1}, \ldots, i_{n}}=\sum_{\left(\beta_{1}, \ldots, \beta_{n}\right) \in B} \lambda_{1 i_{1}}^{\beta_{1}} \ldots \cdot \lambda_{n i_{n}}^{\beta_{n}}
$$

with $1 \leq i_{k} \leq n_{k}$ for $1 \leq k \leq n$.

A first consequence is that every NEPS of integral graphs is integral. It is easily checked that the complete graph $K_{n}$ on $n \geq 2$ vertices has the simple eigenvalue $n-1$ and the eigenvalue -1 with multiplicity $n-1$. Hence NEPS of complete graphs are integral. Using Theorem 2.5, we now readily confirm the following result of [18]:

Proposition 3.2. Every gcd-graph is integral.

An interesting property of a graph is the ability to choose an eigenspace basis such that its vectors have entries from a very small set only. This may be possible only for certain or for all of its eigenvalues. For example, in [9] a construction is given for a basis of the eigenspace of eigenvalue -2 of a generalized line graph whose vectors contain only entries from $\{0, \pm 1, \pm 2\}$.

Imposing an even greater restriction on the admissible entries, we call an eigenspace basis simply structured if it consists of vectors containing only entries from $\{0,1,-1\}$. Accordingly, an eigenspace is considered as simply structured if it has a simply structured basis. Observe that the eigenvalue belonging to a simply structured eigenspace is necessarily integral. 
For a trivial example of a simply structured eigenspace basis, consider a connected $r$ regular graph. Here the all ones vector constitutes a basis of the eigenspace corresponding to the eigenvalue $r$. Moreover, for several graph classes, the eigenspaces corresponding to the eigenvalues 0 or -1 are simply structured, cf. [5],[20],[24].

It is somewhat remarkable if all of the eigenspaces of a graph are simply structured. In [25] is has been shown that Sudoku graphs are NEPS of complete graphs (recall Example 2.9) and admit simply structured eigenspace bases for all eigenvalues. As we shall see, this is true for any NEPS of complete graphs. For this we require the following theorem [11]:

Theorem 3.3. If $X$ and $Y$ are graphs of orders $n$ and $m$ with linearly independent eigenvectors $x^{(1)}, \ldots, x^{(n)}$ and $y^{(1)}, \ldots, y^{(m)}$, respectively, then the $n m$ tensor products

$$
x^{(i)} \otimes y^{(j)} \quad(i=1, \ldots, n ; j=1, \ldots, m)
$$

form a set of linearly independent eigenvectors of any NEPS of $X$ and $Y$. This fact readily extends to NEPS with more factors.

Corollary 3.4. Any NEPS of graphs for which all eigenspaces are simply structured inherits that very property.

Proof. Using the notation of the previous theorem, it is obvious that $x^{(i)} \otimes y^{(j)}$ has only entries from $\{0,1,-1\}$ if the same holds for $x^{(i)}$ and $y^{(j)}$. This remains true for an arbitrary number of factors.

We can now prove the following result:

Proposition 3.5. All eigenspaces of a gcd-graph are simply structured.

Proof. Consider the complete graph $K_{n}, n \geq 2$. The all-ones vector $(1,1, \ldots, 1)$ forms a basis of the eigenspace of eigenvalue $n-1$. A basis of the eigenspace of eigenvalue -1 is formed by the vectors

$$
\begin{array}{lc}
x^{(1)} & =(-1,1,0,0, \ldots, 0,0), \\
x^{(2)} & =(-1,0,1,0, \ldots, 0,0), \\
\vdots & \vdots \\
x^{(n-1)} & =(-1,0,0,0, \ldots, 0,1) .
\end{array}
$$

Thus the result follows from Corollary 3.4 and Theorem 2.5.

\section{Open problems}

Let us conclude with a number of open problems we think are worth investigating in the future:

1. Does every integral Cayley graph over a finite abelian group have a simply structured eigenspace basis for every eigenvalue?

2. Find a small class of integral graphs such that every integral Cayley graph over an abelian group is a NEPS of some graphs of this class.

3. It has been shown by So [26] that integral Cayley graphs over $Z_{p^{\alpha}}, p$ prime, are uniquely determined by their spectrum. Find more groups $\Gamma$ such that cospectral integral Cayley graphs $\operatorname{Cay}\left(\Gamma, S_{1}\right), \operatorname{Cay}\left(\Gamma, S_{2}\right)$ are necessarily isomorphic. 
4. Try to determine or estimate the number $\varrho(n)$ of nonisomorphic gcd-graphs on $n$ vertices. In [18] we showed that for a prime $p \geq 5$ we have $\varrho\left(p^{2}\right)=6$. Observe that $\varrho\left(2^{\alpha}\right)$ is the number of nonisomorphic cubelike graphs on $2^{\alpha}$ vertices, cf. [19].

5. Determine graph invariants for ged-graphs such as connectivity, clique number, and chromatic number, cf. [6], [7].

\section{References}

[1] A. Abdollahi and E. Vatandoost, Which Cayley graphs are integral?, Electron. J. Comb. 16 (2009), R122, 1-17.

[2] A. Abdollahi and E. Vatandoost, Integral quartic Cayley graphs on Abelian groups, Electron. J. Comb. 18 (2011), P89, 1-14.

[3] R. C. Alperin and B. L. Peterson, Integral Sets and Cayley Graphs of Finite Groups, Electron. J. Comb. 19 (2012), P44, 1-12.

[4] K. Balinska, D. Cvetković, Z. Rodosavljević, S. Simić and D. Stevanović, A survey on integral graphs, Univ. Beograd, Publ. Elektrotehn. Fak. Ser. Mat. 13 (2003), 42-65.

[5] S. Barik, S. Fallat and S. Kirkland, On Hadamard diagonalizable graphs, Linear Algebra Appl. 435 (2011), 1885-1902.

[6] M. Bašić and A. Ilić, On the clique number of integral circulant graphs, Appl. Math. Letters 22 (2009), 1406-1411.

[7] M. Bašić and A. Ilić, On the chromatic number of integral circulant graphs, Comp. Math. Appl. 60 (2010), 144-150.

[8] M. Bašić, M. D. Petković and D. Stevanović, Perfect state transfer in integral circulant graphs, Appl. Math. Lett. 22 (2009), 1117-1121.

[9] L. Branković and D. Cvetković, The eigenspace of the eigenvalue -2 in generalized line graphs and a problem in security of statistical databases, Publ. Elektroteh. Fak., Univ. Beogr., Ser. Mat. 14 (2003), 37-48.

[10] D. Cvetković , M. Doob and H. Sachs, Spectra of graphs. Pure and Applied Mathematics, Academic Press, 1980.

[11] D. Cvetković , P. Rowlinson and S. Simić, Eigenspaces of graphs. Encyclopedia of Mathematics and its Applications, Cambridge University Press, 1997, new edition 2008.

[12] D. Cvetković and M. Petrić, Connectedness of the non-complete extended p-sum of graphs, $Z$ b. Rad., Prir-Mat. Fak., Univ. Novom Sadu, Ser. Mat. 13 (1983), 345-352.

[13] G. Hahn and G. Sabidussi (Eds.), Graph Symmetry: Algebraic Methods and Applications, NATO Science Series C, Springer Netherlands, 1997.

[14] F. Harary and A. J. Schwenk, Which graphs have integral spectra?, Lect. Notes Math. 406, Springer Verlag (1974), 45-50.

[15] W. Imrich and S. Klavžar, Product graphs. Structure and recognition., Wiley-Interscience Series in Discrete Mathematics and Optimization, 2000.

[16] D. Kiani, M. M. H. Aghaei, Y. Meemark and B. Suntornpoch, Energy of unitary Cayley graphs and gcd-graphs, Linear Algebra Appl. 435 (2011), 1336-1343.

[17] W. Klotz and T. Sander, Some properties of unitary Cayley graphs, Electron. J. Comb. 14 (2007), R45, 1-12.

[18] W. Klotz and T. Sander, Integral Cayley graphs defined by greatest common divisors, Electron. J. Comb. 18 (2011), P94, 1-15. 
[19] L. Lovász, Spectra of graphs with transitive groups, Period. Math. Hung. 6 (1975), 191-195.

[20] M. Nath and B. K. Sarma, On the null-spaces of acyclic and unicyclic singular graphs, Linear Algebra Appl. 427 (2007), 42-54.

[21] M. Petrić, Connectedness of the generalized direct product of digraphs, Univ. Beograd. Publ. Elektrotehn. Fak. (Ser. Mat.) 6 (1995), 30-38.

[22] H. N. Ramaswamy, C. R. Veena, On the Energy of Unitary Cayley Graphs, Electron. J. Comb. 16 (2009), N24, 1-8.

[23] H. E. Rose, A course in number theory, Oxford Science Publications, Oxford University Press, 1994.

[24] J. W. Sander and T. Sander, On the Kernel of the Coprime Graph of Integers, Integers 9 (2009), $569-579$.

[25] T. Sander, Sudoku graphs are integral, Electron. J. Comb. 16 (2009), N25, 1-7.

[26] W. So, Integral circulant graphs, Discrete Math. 306 (2005), 153-158. 